\title{
Simple method for measuring the porosity, thickness and refractive index of porous silicon, based on the Fabry-Pérot interference spectrum
}

\section{Método simples para medida da porosidade, espessura e índice de refração do silício poroso, baseado no espectro de interferência de Fabry-Pérot}

Tiago Franca Paes ${ }^{1}$, Antônio Fernando Beloto², Ellen Christine de Souza Galvão², Luiz Angelo Berni

\section{ABSTRACT}

This article presents a technique to estimate, nondestructively, the thickness, porosity and refractive index of porous silicon samples. The technique is based on the Fabry-Pérot interference spectrum analyzed by the Reflectometric Interference Fourier Transform Spectroscopy (RIFTS). The technique, also known as SLIM Spectroscopic Liquid Infiltration Method takes into account the infiltration of a liquid with known refractive index inside the pores of the samples.

Keywords: Porous silicon; Porosity; Thickness; Refraction index.

\section{RESUMO}

Este artigo apresenta uma técnica para estimar, de forma não destrutiva, a espessura, a porosidade e o índice de refração de amostras de silício poroso. A técnica baseia-se no espectro de interferência de Fabry-Pérot analisada com o método de Espectroscopia de Interferência Reflectométrica por Transformada de Fourier. A técnica, também conhecida como SLIM - Espectroscopia por Infiltração de Líquidos, leva em conta a infiltração de um líquido com um índice de refração conhecido, nos poros das amostras.

Palvras-chave: Silício poroso; Porosidade; Espessura; Índice de refração 


\section{INTRODUCTION}

The porous silicon (PSi) formation process is rather complex and depends on chemical and physical factors. The composition of the electrolyte and its concentration, current applied, etching time, temperature, luminous intensity, type of dopants and the doping density of the silicon substrate are some factors that influence the morphology such as size and depth of pores and type of porous structure ${ }^{(1)}$. In general, the porosity increases with increasing applied current density and decreases with the increase in HF concentration in the electrolyte, in addition, it depends on the pore characteristics and can vary substantially due to doping density of the substrate ${ }^{(2)}$. Generally, the pore size increases with the concentration of dopants for p-type silicon and decrease with the concentration of dopant for n-type.

To determine porosity and thickness, many techniques are available and some are destructive or time-consuming. The gravimetric method, for example, is destructive because relates the mass of the sample before and after pore formation, removing the porous layer using a base solution ${ }^{(3)}$. The X-ray diffraction characterization method, as reflectivity of grazing incidence configuration, is widely used in structural analysis of many materials ${ }^{(4)}$. Despite being a non-destructive measurement technique, it requires a long time to measure.

Considering the PSi as a thin film, when a light beam strikes the surface of a material, the light may reflect, refract, scatter and/or be absorbed. In this way, the Fabry-Pérot interference principle can be used to analyze spectral reflectance patterns and estimate morphological characteristics of the porous layer as porosity and thickness.

\section{FABRY-PEROT INTERFERENCE PRINCIPLE FOR PSi}

In the interaction of light with a porous silicon sample, it should be considered a system composed by air, PSi layer and the silicon wafer. In this case, we will discard the fraction of absorbed and scattered radiation by the interface for having negligible intensities. Assuming that the incident angle is normal to the surface, from Euclid's law and Snell's law of reflection and refraction respectively we can ignore the angular dependence of reflection. From the optical properties of reflection and transmission of radiation by different PSi interfaces, and the wave behavior of electromagnetic radiation, it is possible to observe, in this system, constructive and destructive interference fringes of the transmitted and reflected radiation ${ }^{(5)}$. The measurement of the PSi reflectance spectrum shows a series of interference fringes corresponding to constructive and destructive interferences. As an example, Fig. 1 (A) shows the Fabry-Pérot interference fringes from the reflectance spectrum measurement of the PSi, while Fig. 1 (B) is a graph of the integers numbers corresponding to all the fringes peak as a function of the frequency $(1 / \lambda \mathrm{p})$ at which each fringe appears in the spectrum following the Eq. 1:

$$
m \lambda_{\max }=2 n_{P S i} L
$$

where $\lambda_{\max }$ is wavelength of the fringe maximum, $n_{P_{s i}}$ is the average refractive index of the PSi layer, $L$ is the thickness of PSi layer and $m$ is the integer number of spectral order of the fringe ${ }^{(6)}$. The factor of 2 is due to the optical path taken by the light source PSi - detector, where the source and the detector are configured in the same position (normal incidence). Therefore, the term $2 n_{P S i} \mathrm{~L}$ is the optical path length, also called Effective Optical Thickness $-\mathrm{EOT}^{(7)}$.

A simple method to calculate the EOT of PSi layer is by measuring the reflectance spectrum showed on Fig. 1. Each maximum peak $(P)$ follows the Eq. 1 as $P=2 n_{P S i} \mathrm{~L}\left(1 / \lambda_{\max }\right)+b$. In this example, the $P$ is enumerated from 0 to 12 and is related to the frequency. The $m$ value is calculated from the straight line equation and given from 19 to 31 and the EOT gives $17820 \mathrm{~nm}$.

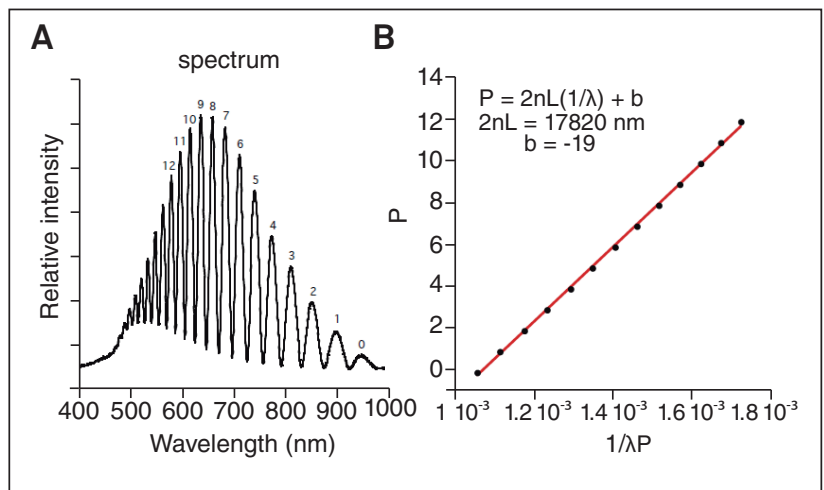

Figure 1: Simple Method to calculate 2nPSiL from the Fabry-Pérot interference spectrum: (A) Measurement of reflectance spectrum of a PSi sample listed some fringes. (B) Fringes as a function of frequency for each fringe $1 / \lambda p$.

\section{BRUGGEMAN EFFECTIVE MEDIUM APPROXIMATION MODEL TO CALCULATE THE REFRACTIVE INDEX OF PSI SAMPLES}

The refractive index of the PSi is composed by a portion of silicon refractive index (porous skeleton) and other portion is the medium filling the pores which can be the refractive index of air or any other substance that can completely fill the pores. The Bruggeman Effective Medium Approximation can estimate porosity and average refractive index of PSi layer using the Eq. $2^{(8)}$,

$$
(1-P) \frac{\left(n_{S i}^{2}-n_{P S i}^{2}\right)}{\left(n_{S i}^{2}+2 n_{P S i}^{2}\right)}+(P) \frac{\left(n_{P o r e}^{2}-n_{P S i}^{2}\right)}{\left(n_{P o r e}^{2}+2 n_{P S i}^{2}\right)}=0
$$

where, $P$ is the porosity, $n_{s i}$ is silicon refractive index, $n_{\text {pore }}$ is the refractive index of the substance that fills the pores, for example, the air or a known alcohol index, and $n_{P S i}$ is the average refractive index of the composite PSi layer, considering both components (skeleton and pore). The Eq. (2) can be solved for the refractive index of the PSi composite layer ${ }^{(6)}$ : 
Based on Eqs. 1, 2 and 3 it is possible to estimate the porosity and the thickness of PSi relating two different $n_{\text {pore }}$, for example, air and a known alcohol. This method for estimating the thickness and porosity of the PSi is called Spectroscopic Liquid Infiltration Method - $\operatorname{SLIM}^{(9)}$.

\section{SPECTROSCOPIC LIQUID INFILTRATION METHOD - SLIM}

Figure 2 shows the schematic diagram of the SLIM system mounted in the laboratory at INPE. The samples were illuminated perpendicularly to the PSi sample with a light source using a halogen lamp GetSpec, model GetLight 3093 SET. The sample was fixed on a table with height and horizontal adjustments to improve the reflected signal and scan the PSi surface. A bifurcated fiber optic Thorlabs, model RP20, connected to the light source and the Oceanoptics spectrometer, model USB2000 facilitates the signal acquisition and sample illumination by being in the same position on the sample. The spectral range of the spectrometer is from 350 to $1000 \mathrm{~nm}$, enough to perform the measurement of the reflectance. A first side mirror at the same position of the sample to be measured was used as a measurement reference. For each sample, the reflectance spectrum was measured from the dry sample first and after with a liquid filling the pores. Typically, the liquid used is absolute ethanol at a concentration of $99.8 \%$ with refractive index $\mathrm{n}=1,3611$ for $\lambda=700 \mathrm{~nm}$. Measuring the reflectance in this two ways allows us to estimate the relative refractive index, the EOT of PSi and calculate its porosity and thickness. Figure 3 illustrates the SLIM measurement modes i.e. with two different refractive indices.

The Reflectometric Interference Spectroscopy allows to obtain the EOT $(2 \mathrm{~nL})$ of the PSi directly from Fourier transform (FFT) of reflectance spectra obtained from two different medias, where the peak position given by the FFT is equal to $2 \mathrm{~nL}$ and the amplitude

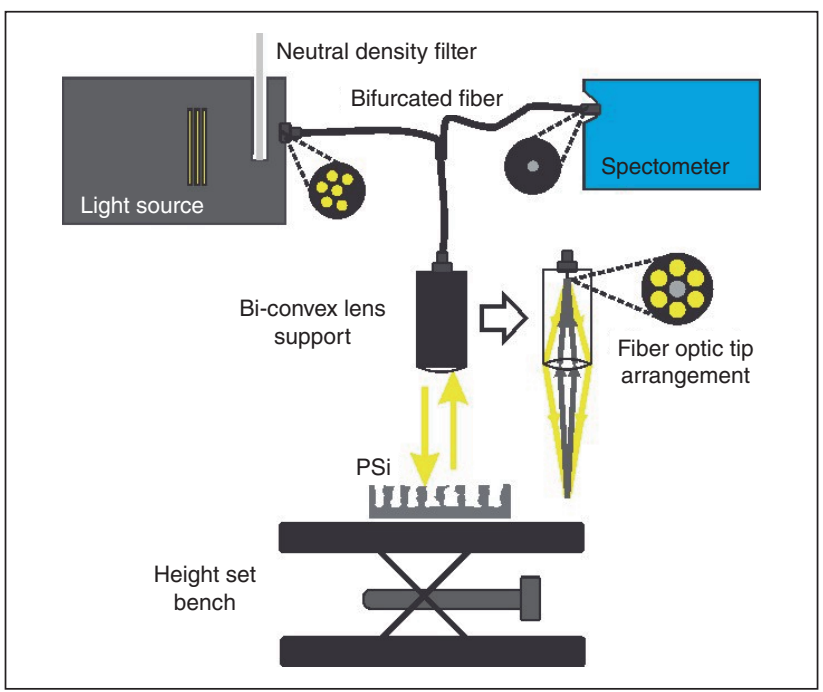

Figure 2: Schematic diagram of the SLIM optical set-up. is related to the refractive index of PSi. Figure 4 shows the Fourier transform from a typical reflectance spectrum.

Each measured reflectance spectrum determines the PSi EOT $(2 \mathrm{~nL})$. Defining the parameters measured EOT in air and EOT in liquid and relate to Eqs. 1, 2 and 3, it can provide an estimate of the porosity and thickness. Computer codes that implement the calculation are available at http://sailorgroup.ucsd.edu/ software ${ }^{(6)}$.

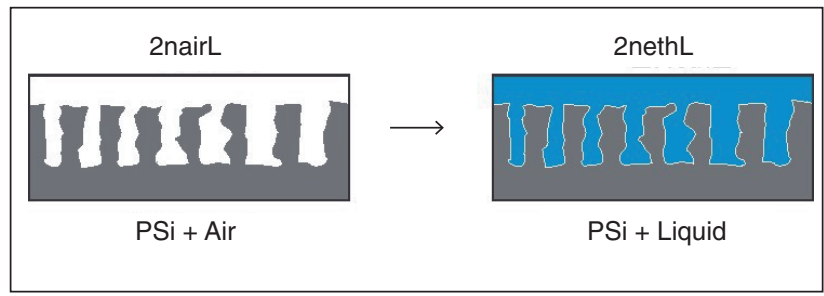

Figure 3: Different refractive indices of media filling the pores to measure the PSi reflectance media of different.

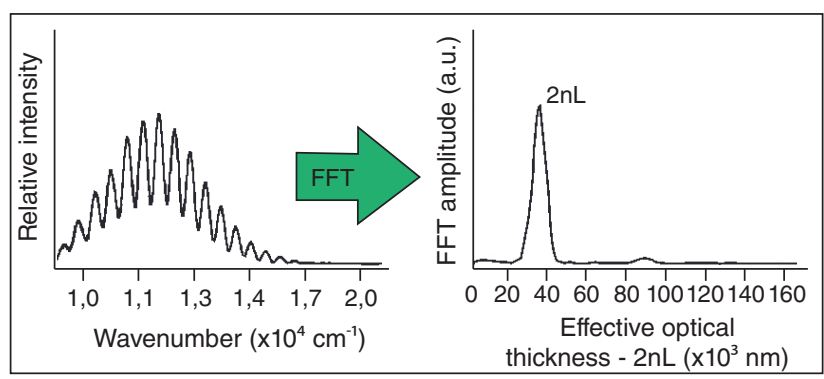

Figure 4: Reflectometric Interference spectrum by FFT (RIFTS)

\section{SAMPLES PREPARATION}

In this work were used highly doped boron (p-type) silicon wafers as substrate with resistivity from 0.01 to $0.02 \Omega \mathrm{cm}$, crystallographic orientation (100) and thickness between $375-425 \mu \mathrm{m}$. Two solutions containing HF (48\%) and ethanol (99.5\%) were used with different ratios: $3: 1$ and 1:1 by volume concentration. Table 1 shows the preparation conditions of PSi samples, identifying those prepared only for gravimetric measures, because it is a destructive measure.

\section{RESULTS AND DISCUSSION}

The gravimetric study aimed to estimate the porosity and thickness of the PSi layer, by mass calculation of each sample in different stages of the etching process. As a destructive technique, the reproducibility of similar samples becomes a challenge when they are measured and characterized in others equipment. Table 2 reports the values obtained from the masses measurement of the samples.

Analyzing the results obtained by the gravimetric method, we can say that, considering the etching parameters adopted at work (Table 1), the thickness increases with the etching time and the current density, regardless of the concentration of HF solution. 
During the acquisition of the reflectivity spectrum of X-ray grazing incidence, the beam undergoes a total reflection at angles less than the critical angle, because of PSi refractive index. For angles greater than the refracted beam in the material, it causes a sharp drop in the intensity of the measured signal. In the case of porous silicon, whose surface consists of crystallites and pores, the reflectivity behaves as a diffuse scattering which, in this case, hinders the observation of interference fringes ${ }^{(10)}$. Figure 5 shows the reflectivity spectra of X-ray grazing incidence, along with a sample silicon substrate before forming PSi in linear scale.

From the spectra, it is possible to find the critical angle of the monocrystalline silicon substrate and it is measured by the half intensity in spectra X-ray reflectivity curve, where $\omega_{c}=0.227^{\circ}$, that is very close to the theoretical value given as $0.224^{\circ}$ for incident equal to 1.55 and the energy of the $\mathrm{X}$-ray beam equal to $8 \mathrm{KeV}$. Figure 5 shows that the critical angles of the PSi samples are smaller than that found for the silicon substrate. That is explained because of the lower density of PSi layer ${ }^{(11)}$. Table 3 shows the critical angles values of the PSi samples.

Considering these values, the porosity of each measured sample could be estimated based on the equation $\omega_{c}=\sqrt{ } 2 \delta$ where $\omega_{c}$ is related with the dispersion $(\delta)$ of the incident radiation over the material surface, which is function of the refractive index ${ }^{(10)}$. Therefore, the porosity could be defined by $\delta \alpha P_{x}$, using the expression: $P=1-\left(\omega_{\mathrm{cPsi}} / \omega_{\mathrm{csi}}\right)^{2(11)}$. The results are shown on Table 4 .

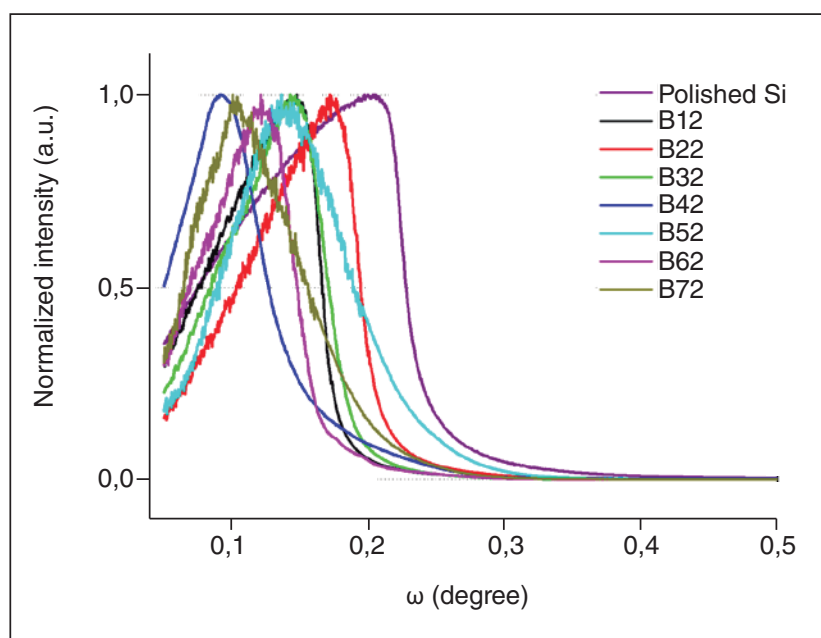

Figure 5: Spectra X-ray reflectivity at grazing incidence angle in linear scale.

Table 1: Identification of main samples and their respective etching parameters

\begin{tabular}{|c|c|c|c|c|}
\hline Sample name & $\begin{array}{c}\text { Solution concentration } \\
{[\mathbf{v o l}]}\end{array}$ & $\begin{array}{c}\mathbf{J} \\
{\left[\mathbf{m A} / \mathbf{c m}^{2}\right]}\end{array}$ & Time [min] & Technique \\
\hline B12 & $1: 1$ & 30 & 10 & SLIM/X-ray \\
\hline B13 & $1: 1$ & 30 & 10 & Gravimetric \\
\hline B22 & $3: 1$ & 30 & 10 & SLIM/X-ray \\
\hline B23 & $3: 1$ & 30 & 10 & Gravimetric \\
\hline B32 & $3: 1$ & 300 & 1 & SLIM/X-ray \\
\hline B33 & $3: 1$ & 300 & 1 & Gravimetric \\
\hline B42 & $1: 1$ & 300 & 1 & SLIM/X-ray \\
\hline B43 & $1: 1$ & 300 & 10 & Gravimetric \\
\hline B52 & $3: 1$ & 80 & 10 & SLIM/X-ray \\
\hline B53 & $3: 1$ & 80 & 10 & Gravimetric \\
\hline B62 & $1: 1$ & 80 & 10 & SLIM/X-ray \\
\hline B63 & $1: 1$ & 80 & 20 & Gravimetric \\
B72 & $1: 1$ & 80 & 20 & SLIM/X-ray \\
B73 & $1: 1$ & 80 & Gravimetric \\
\hline
\end{tabular}

Table 2: Characteristics values of porosity and thickness obtained by gravimetric technique.

\begin{tabular}{|c|l|l|l|l|l|l|l|}
\hline Samples & B13 & B23 & B33 & B43 & B53 & B63 & B73 \\
\hline Porosity $(\%)$ & 41,9 & 32,3 & 54,6 & 79,3 & 48,8 & 58,9 & 65,5 \\
\hline Thickness $(\mu \mathrm{m})$ & 22,0 & 26,1 & 15,6 & 10,6 & 47,0 & 41,3 & 76,2 \\
\hline
\end{tabular}

Table 3: Values of critical angles of PSi samples obtained from the spectral analysis of $x$-ray reflectivity for grazing incidence.

\begin{tabular}{|c|c|c|c|c|c|c|c|c|}
\hline Samples & B12 & B22 & B32 & B42 & B52 & B62 & B72 \\
\hline (radians) & 0,166 & 0,195 & 0,171 & 0,127 & 0,188 & 0,148 & 0,156 \\
\hline
\end{tabular}

Table 4: Porosity of PSi samples obtained from the spectral analysis of $x$-ray reflectivity for grazing incidence.

\begin{tabular}{|c|c|c|c|c|c|c|c|}
\hline Samples & B12 & B22 & B32 & B42 & B52 & B62 & B72 \\
\hline Thickness $(\mu \mathrm{m})$ & 46,7 & 26,5 & 43,2 & 68,9 & 31,8 & 57,7 & 52,9 \\
\hline
\end{tabular}


Comparing the results with the gravimetric technique, the average of the standard deviation is $13.5 \%$. The feasibility of this technique is that it is a non-destructive method. However, for samples that have a high degree of scattering of the incident beam, the result becomes more divergent from the actual value.

From SLIM technique, the optical thickness of the PSi sample is determined from the application of the Fourier transform of the PSi interferometric reflectance spectra (Fabry-Perot interference spectrum) filled the pores with air and ethanol. The differences between the spectra are attributed on the variation of EOT due to the change of the medium refractive index inside the pores, with the assumption that all voids were filled equally. Figure 6 shows an interferometric reflectance spectra of a typical PSi pores filled with air and ethanol. The Fourier Transform of the spectra over the Fig.6 defines the RIFTS as shown in Fig. 7.

RIFTS provides the optical thickness (2nL) of PSi by the peak position, and it is possible to estimate the refractive index, porosity and thickness of the samples using the Eqs. 1 and 2. The calculated values are shown in Table 5. The program used to calculate the porosity and the thickness from the optical thickness values $(2 \mathrm{~nL})$ from both spectra, filled pores with air and ethanol, can also estimate the value of the refractive index of the porous layer if the thickness is known, e.g. SEM images, so also the porosity considering the new calculated index value.

To compare the results of using other SLIM procedure, the Table 6 inform the values of PSi refractive indexes for $\lambda=680 \mathrm{~nm}$, and porosity values from the thickness obtained by the SEM images of the samples.

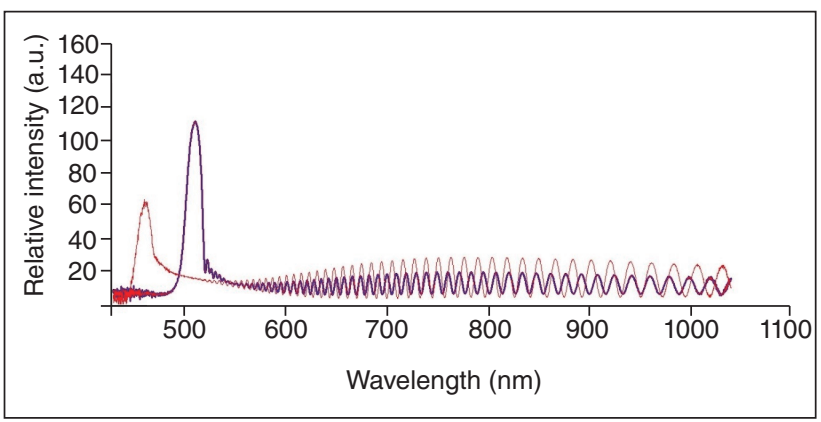

Figure 6: Interferometric reflectance spectra of a typical PSi pores filled with air and ethanol.
Analyzing the thickness values found by the SLIM technique and compare to other techniques as the SEM images and gravimetric method, the divergence between those techniques is $\pm 5.11 \%$. Comparing the calculated values related to porosity using this technique and the porosity estimated by gravimetric technique, there is a disparity of $26.4 \%$. This value can be considered a little high, as it represents about $1 / 4$ of uncertainty for the values obtained. Comparing the values of porosity encountered by the X-ray grazing incidence technique, the deviation is $21.7 \%$. This value can be explained by the different sensibilities of each technique explored. The results of the refractive index estimated by the SLIM method are close than expected when compared to other references(12). Assume the refractive index of the crystalline silicon is approximately 3.8 at $\lambda=700 \mathrm{~nm}$, the index of PSi should be smaller than the index of the crystalline silicon.

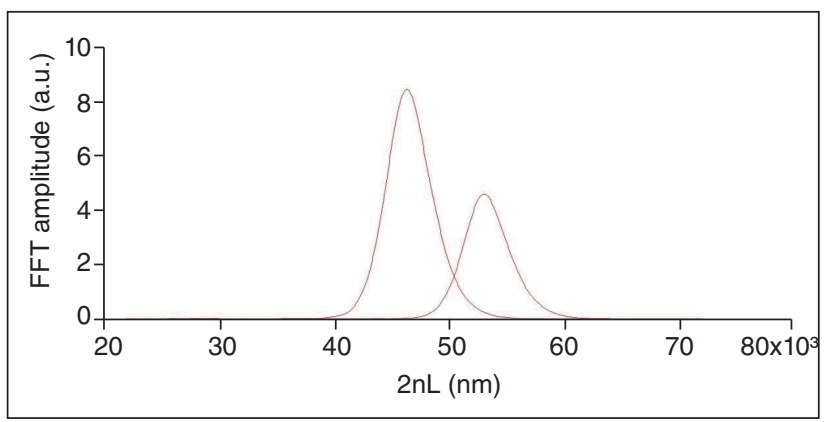

Figure 7: Reflectometric Interference Spectroscopy by FFT obtained from interferometric reflectance spectra as showed in Fig. 6.

\section{CONCLUSION}

In this work a nondestructive technique to estimate thickness, porosity and refractive index of porous silicon samples is presented. These techniques are based on the Fabry-Pérot interference spectrum analyzed by the Reflectometric Interference Fourier Transform Spectroscopy (RIFTS) and liquid infiltration spectroscopy (SLIM). In the case of SLIM system, a factor that can directly affect the values associated with the liquid infiltration into the pores occur when they are not filled completely. This is due to two possible reasons: the pores may be physically closed due to structural variation that occurs by oxidation process or the

Table 5: Values of the porosity and thickness of PSi samples obtained by SLIM.

\begin{tabular}{|c|c|c|c|c|c|c|c|c|}
\hline Samples & B12 & B22 & B32 & B42 & B52 & B62 & B72 \\
\hline Porosity $(\%)$ & 26,2 & 8,8 & 43,9 & 80,7 & 33,3 & 47,9 & 54,1 \\
\hline Thickness $(\mu \mathrm{m})$ & 23,8 & 26,1 & 16,1 & 11,3 & 43,4 & 38,8 & 76,2 \\
\hline
\end{tabular}

Table 6: Refractive index and porosity of PSi samples obtained by SLIM and the thickness by SEM images.

\begin{tabular}{|c|c|c|c|c|c|c|c|c|}
\hline Samples & B12 & B22 & B32 & B42 & B52 & B62 & B72 \\
\hline Porosity (\%) & 26,5 & 8,6 & 43,1 & 77,2 & 30,4 & 43,3 & 51,3 \\
\hline PSi Refractive index & 3,1 & 2,9 & 3,0 & 3,5 & 2,5 & 2,3 & 2,6 \\
\hline
\end{tabular}


surface tension of the liquid can be relatively high, which would prevent infiltration of liquid into smaller pores ${ }^{(13)}$. However, this technique becomes quite feasible when compared with the others, because it is not a destructive technique, the result is displayed immediately and requires no sample preparation. The measured values of porosity, thickness and refractive index of PSi samples compared to others characterization techniques suggests the Fabry-Perot interference spectrum a convenient method to estimate those proprieties of PSi. Moreover, we suggest to compare this method using others liquids with different refractive index to minimize the effect of surface tension by the pores structure.

\section{REFERENCES}

1. ZHANG, X.G., Electrochemistry of Silicon and its Oxide. New York: Kluwer Academic/Plenum Publishers, 2001.

2. LEHMANN, V., Electrochemistry of silicon: instrumentation, science, materials and applications. Wiley-VCH, 2002. v. 1, p. 286. ISBN 3-527-29321-3.

3. SCHUBERT, M., Gravimetric determination of the porosity of porous silicon. University of Western Australia, Australia, 2010.

4. GOUDEAU, P. et al. ,X-ray small-angle scattering analysis of porous silicon layers. Journal of Applied Physics, v. 66, n. 2, p. 625-628, 1989.
5. MACLEOD, H. A., Thin film optical filters. 3. Eb. CRC Press, 2001 668 p. ISBN: 1420033239,9781420033236

6. SAILOR, M. J., Porous silicon in practice: preparation characterization and applications. John Wiley \& Sons, 2012.

7. PACHOLSKI, C. et al., Biosensing using porous silicon doublelayer interferometers: reflective interferometric Fourier transform spectroscopy. Journal of the American Chemical Society, v. 127, n. 33, p. 11636-11645, 2005.

8. GARAHAN, A. et al., Effective optical properties of absorbing nanoporous and nanocomposite thin films. Journal of applied physics, v. 101, n. 1, p. 014320, 2007.

9. SEGAL, E. et al., Confinement of thermoresponsive hydrogels in nanostructured porous silicon dioxide templates. Advanced Functional Materials, v. 17, n. 7, p. 1153-1162, 2007.

10. PARRAT, L. G., Surface studies of solids by total reflection of X-ray. Physical Review, v.95, n. 2, p.359-369, 1954.

11. ABRAMOF, P. G. et al., X-ray investigation of nanostructured stain-etched porous silicon. Journal of applied physics, v. 99, n. 2, p. 024304, 2006.

12. THEISS, W. ; HILBRICH, S., Refractive index of porous silicon In: Canham L (ed) Properties of porous silicon. Institution of Engineering and Technology, London, pp 223-228, 1997.

13. PAP, A. E. et al., Optical properties of porous silicon. Part III: Comparison of experimental and theoretical results. Optical Materials, v. 28, n. 5, p. 506-513, 2006. 